\title{
Effect of Rubbing on the Distribution of Topically Applied Drugs into the Hair Follicles
}

\author{
Akinari Abe ${ }^{a, b}$ Hiromichi Suzuki, ${ }^{a}$ Miyuki Saito,${ }^{a}$ Hiroaki Todo ${ }^{a}$ and Kenji Sugibayashi ${ }^{*}, a$ \\ ${ }^{a}$ Graduate School of Pharmaceutical Sciences, Josai University; 1-1 Keyakidai, Sakado, Saitama 350-0295, Japan: \\ and ${ }^{b}$ Research \& Development Headquarters Self-Medication, Taisho Pharmaceutical Co., Ltd.; 1-403 Yoshinocho, \\ Kita-ku, Saitama 331-9530, Japan.
}

Received January 21, 2020; accepted May 2, 2020

Rubbing actions are often conducted to apply topical formulations onto the skin. Although rubbing was found to increase the skin permeation of drugs, few reports have revealed whether rubbing enhanced either drug permeation through the stratum corneum (SC) or hair follicles (HFs) pathways, or through both. In the present study, we investigated the effects of rubbing on caffeine (CAF) distribution in the SC and HFs. The effect of rubbing direction on the skin penetration of CAF was also investigated. The skin concentration of CAF and its cumulative permeation amount were increased clearly by rubbing. More than six times higher CAF concentrations in the viable epidermis and dermis were observed $5 \mathrm{~min}$ after rubbing application compared with no rubbing. On the other hand, slightly increased CAF concentrations were observed in the SC, suggesting that CAF was delivered through the HF pathway by rubbing. Rubbing against the natural hair direction provided the highest skin permeation as well as skin concentrations. Changes in the morphology of the HF opening area might be related to the enhancement effect. These results may provide useful information to understand the effect of rubbing on the skin permeation of applied drugs.

Key words rubbing application; hair follicle targeting; skin permeation; drug disposition

\section{Introduction}

In vitro skin permeation experiments are important to evaluate the safety and efficacy of topically applied drugs and exposure to such compounds. Understanding of the permeation pathway of topically applied drugs through the stratum corneum (SC) and hair follicles (HFs) is useful for the development of topical formulations. In in vitro skin permeation experiments, formulations are usually applied to excised skin using a dispenser or a spatula to spread the formulation uniformly on the application area. On the other hand, in practical use, semi-solid formulations such as ointments, lotions, and creams are applied using the fingers with rubbing. Thus, the application methods of formulations can differ between in vitro skin permeation experiments and practical use. This difference may cause large differences in the skin permeation and concentrations of topically applied drugs.

Several reports have already found that rubbing of topical formulations enhanced the skin permeation of the drugs contained in them. ${ }^{1-4)}$ The amounts of drug distributed to the SC and HFs are thought to be closely related to the skin permeability of the applied drugs. Therefore, it is important to understand changes in drug disposition in either the SC or HFs or both. Drug disposition in the SC can be determined using the tape stripping (TS) method, ${ }^{5)}$ and the TS method is now utilized in bioequivalence assessments of generic drugs for topically applied formulations to the skin. On the other hand, methods to evaluate drug disposition in HFs are not well established. This may be attributed to lower hair follicle density $\left(0.1 \%\right.$ the total skin area) ${ }^{6)}$ However, HF pathway is related to the early phase of skin permeation ${ }^{7)}$ and the early detection of topically applied drug in the blood. ${ }^{8,9)}$

In our previous study, we reported that the cyanoacrylate biopsy (CB) method can be used to measure the drug concen- trations in HFs by obtaining a highly reproducible HF replica with an average height of $175 \mu \mathrm{m} .{ }^{10)}$ The CB method can successfully collect drug distributed in the shallow area in HFs. Therefore, the combination of TS and CB methods can separately analyze drug distribution profiles in the SC and HFs.

Increased drug disposition into HFs by rubbing application might cause more rapid detection in the blood and higher blood concentrations of applied drugs. Therefore, the effects of rubbing on the drug disposition into HFs as well as the SC and skin permeation were investigated to develop semi-solid dosage formulations with a higher effect and better safety in the present study. Normally, rubbing application is performed without awareness of the direction of growth of hairs. Because rubbing application may induce a histological change in the opening area of HFs, the amount of drug distribution in HFs may be changed by the rubbing direction. Therefore, in the present study, the effect of rubbing direction on drug disposition into the SC and HFs was also investigated.

A model hydrophilic drug, caffeine (CAF), was used in this study, and the effect of CAF distribution in skin and its skin penetration was investigated after topical application with different rubbing directions. In addition, Fluoresbrite ${ }^{\circledR}$, yellowgreen nanospheres, was used to clarify the effect of rubbing direction on HF disposition by visible observation.

\section{Experimental}

Materials CAF (M.W.: 194.2, $\left.\log K_{\mathrm{o} / \mathrm{w}}:-0.12\right)$ obtained from FUJIFILM Wako Pure Chemical Corporation (Osaka, Japan). Aron Alpha ${ }^{\circledR}$ (multi-purpose type, Toagosei Co., Ltd., Tokyo, Japan) and cellophane tape (Standard D-Squame Discs, CuDerm Corporation, Dallas, TX, U.S.A.) were used for the $\mathrm{CB}$ and TS method, respectively. Teflon mesh (ETFE Teflon 40 mesh) was obtained from Clever Co., Ltd. (Toyohashi, 
Aichi, Japan). Fluoresbrite ${ }^{\circledR}$ yellow-green carboxylate microspheres ( $50 \mathrm{~nm}$ average diameter) were purchased from Polysciences Inc. (Warrington, PA, U.S.A.) as model nanosheres. Frozen edible pig ear skins (17-23 weeks of age) were purchased from the National Federation of Agricultural Cooperative Associations (Ibaraki, Ibaraki, Japan). These skins were stored at $-80^{\circ} \mathrm{C}$ before the in vitro experiments. All other reagents and solvents of the highest available purity and used as purchased.

Particles Distribution in Skin after Rubbing Application Porcine ear skin with the underlying cartilage was used as described. ${ }^{9)}$ Briefly, after thawing the frozen skin tissue at $32^{\circ} \mathrm{C}$, the skin tissue was carefully washed with purified water. Then, the hair-shaved skin with an electric shaver was set into a Franz-type diffusion cell (effective diffusion area: $1.77 \mathrm{~cm}^{2}$ ). After $1.0 \mathrm{~h}$ hydration process with $1.0 \mathrm{~mL}$ purified water in an incubator (MCO-18AIC, Sanyo Electric Co., Ltd., Osaka, Japan) at $32^{\circ} \mathrm{C}$ and $80 \%$ relative humidity (RH) conditions, the purified water was removed from the diffusion cell.

Fluoresbrite $^{\circledR}$ suspension (conc. 2.5\% (w/w); suspended in distilled water) was applied on the excised porcine ear skin in the Franz-type diffusion cell with or without rubbing action. Rubbing action was conducted using a rubber-encased forefinger for $30 \mathrm{~s}$ with a pressure of $1.87-3.12 \mathrm{~N} / \mathrm{cm}^{2}{ }^{1)}$ ) During the rubbing action, the loading force from a finger on the skin was measured using a balance so as not to exceed the designated range. One-time rubbing action was performed for $0.5 \mathrm{~s}$, and the action was continued for $30 \mathrm{~s}$. Three different rubbing directions were examined in the present study; rubbing application in a circular pattern on the skin (drHF), rubbing application along the direction of hair growth (alHF), and rubbing application against the direction of hair growth (agHF). Hair growth directions were confirmed visually before application with the use of a digital microscope (VHX-5000, Keyence Co., Ltd., Osaka, Japan). A without-rubbing action control was conducted by applying the compound homogeneously using a spatula to spread out the compound on the skin surface. The skin was washed three times with $1 \mathrm{~mL}$ of purified water to remove the applied suspension 5 min after application, and the skin was embedded in Tissue-Tek ${ }^{\circledR}$ OTC compound (Sakura Finetek Japan Co., Ltd., Tokyo, Japan). Horizontal skin sections were obtained using a cryostat (CM3050S; Leica, Wetzlar, Germany) with $10 \mu \mathrm{m}$-thickness. The prepared skin sections were observed using a fluorescence microscope (CK40; Olympus Corp., Tokyo, Japan) to evaluate the penetration of the applied compound by rubbing action.

In Vitro Skin Permeation Experiment The in vitro permeation profiles of CAF were obtained using excised porcine ear skin. The excised porcine ear skin membranes were mounted in a Franz-type diffusion cell. Then, $20 \mu \mathrm{L}$ of CAF solution $(100 \mathrm{mM}$, prepared with purified water) was applied to the SC side and purified water was applied to the dermal side (receiver cell volume: $6.0 \mathrm{~mL}$ ). Just after the application of CAF solution, three different rubbing treatments were performed at a pressure of $1.87-3.12 \mathrm{~N} / \mathrm{cm}^{2}$ using a rubberencased forefinger for $30 \mathrm{~s}$. Permeation experiments were conducted at $32^{\circ} \mathrm{C}$ for $2 \mathrm{~h}$, during which the receiver solution was stirred continuously with a star-head-type magnetic stirrer. As a without-rubbing application control, the applied CAF solution was spread out homogeneously with a spatula on the skin surface. At predetermined times, $0.5 \mathrm{~mL}$ of the receiver solution was sampled, and the same volume of purified water was added to keep the volume constant.

CAF Distribution in Skin The same procedure as section, "Particles Distribution in Skin after Rubbing Application," was applied for CAF application. After removal of excess water from the skin surface after finishing the hydration process, rubbing application of $100 \mathrm{mM}$ CAF solution $(20 \mu \mathrm{L})$, which was prepared with purified water, was conducted using a rubber-encased forefinger for $30 \mathrm{~s}$ with a pressure of $1.87-3.12 \mathrm{~N} / \mathrm{cm}^{2}$. The CAF solution was spread out homogeneously with a spatula on the skin surface as a withoutrubbing action control.

CAF Distribution of in SC and HFs After finishing the application period (5 or $120 \mathrm{~min}$ ), the skin was thoroughly washed three times with $1 \mathrm{~mL}$ of purified water. Distributed CAF in the SC and HFs were collected by TS and CB methods, respectively. ${ }^{10)}$ Briefly, twenty successive TS procedures were performed to collect CAF in the SC layers from the applied site. Then, after measurement the weight of the SC removed, CAF was extracted in $1.0 \mathrm{~mL}$ of ethanol in a glass vial for $1 \mathrm{~h}$ from the SC layers.

Cyanoacrylate liquid was applied onto the TS treated area, and then a sheet of cellophane tape was placed onto the area. The cellophane tape was removed to collect the cured cyanoacrylate (HF replica) from the skin. CAF was extracted from the HF replica in $1.0 \mathrm{~mL}$ of methanol in a glass vial.

CAF Distribution of in Viable Epidermis/Dermis (VED) The weight of the obtained skin piece was measured after finishing the TS and $\mathrm{CB}$ procedures. Then, the skin was minced with scissors and homogenized $\left(4^{\circ} \mathrm{C}, 5 \mathrm{~min}\right)$ with $2.0 \mathrm{~mL}$ of methanol for $2.5 \mathrm{~min}$ using a homogenizer (Polytron PT-MR 3000; Kinematica Inc., Littau, Switzerland). After centrifugation $\left(21200 \times \boldsymbol{g}, 4^{\circ} \mathrm{C}, 15 \mathrm{~min}\right), 200 \mu \mathrm{L}$ of the supernatant was mixed with the same volume of methanol and centrifuged again using the same parameters.

Detection of CAF Concentration CAF concentrations in the samples were detected using an HPLC system (Prominence; Shimadzu, Kyoto, Japan) which was reported previously ${ }^{10)}$ After centrifugation of the samples at $21500 \times \boldsymbol{g}$ and $4^{\circ} \mathrm{C}$ for $5 \mathrm{~min}$, the supernatant $(20 \mu \mathrm{L})$ was added to the same volume of acetonitrile. Then, the solution was injected into the HPLC system. Analysis was performed with an Inertsil-ODS-3 column $\left(5 \mu \mathrm{m}, 150 \times 4.6 \mathrm{~mm}^{2}\right.$ i.d.; GL Science, Kyoto, Japan) at $40^{\circ} \mathrm{C}$ using $0.1 \%$ phosphoric acid-acetonitrile $(10: 90, \mathrm{v} / \mathrm{v})$ as mobile phase (the flow rate $1.0 \mathrm{~mL} / \mathrm{min}$ ). The detection was performed at UV $254 \mathrm{~nm}$. CAF concentration $\left(\%\right.$ dose $\left./ \mathrm{cm}^{2}\right)$ in HFs was corrected using the total opening HF area in the CAF application area, whereas that in the SC was corrected using the neat SC area calculated from the subtraction of the total opening HF area from the CAF application area. The total amount of CAF in the skin was calculated using the sum of collected CAF from the SC layer, HF replica, and VED.

Calculation of Enhancement Ratio The enhancement ratio $(E R)$ for the cumulative amount of CAF permeated $(Q)$ and the skin concentration from the rubbing actions were calculated by dividing that obtained from the without-rubbing control application at the same time points. ERp and ERc were used to distinguish $E R$ values from that obtained from in vitro skin permeation and skin concentration experiments, respectively.

Statistical Analysis Statistical analysis was carried out 
using unpaired Student's t-test with $\mathrm{JMP}^{\circledR}$ Pro (ver. 14.1.0, SAS Institute, Cary, NC, U.S.A.). A $p$ value less than 0.05 was considered significant.

\section{Results}

Particles Distribution in Skin Fluoresbrite ${ }^{\circledR}, 50 \mathrm{~nm}$ yellow-green nanospheres, was used to evaluate the penetra-

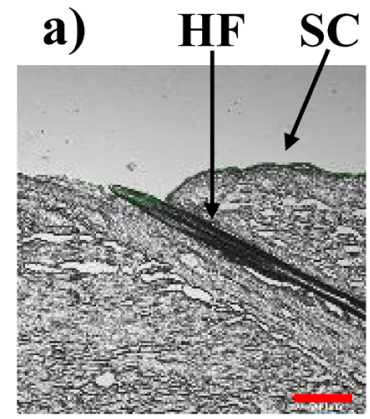

c)

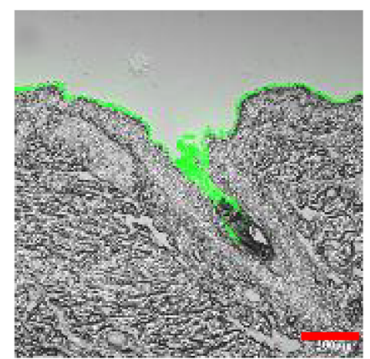

b)

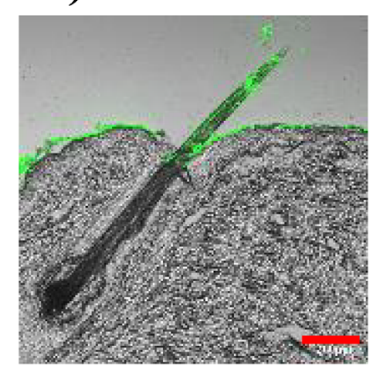

d)

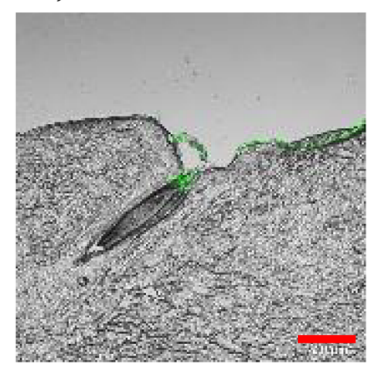

Fig. 1. Distribution of Fluoresbrite ${ }^{\circledR}$ in the Skin after Application withor without-Rubbing Action

(a): without rubbing, (b) drHF, (c) agHF, and (d) alHF. Green color indicates the distribution of fluorescence from Fluoresbrite ${ }^{\circledR}$. HF: hair follicle, SC: stratum corneum. Bar: $200 \mu \mathrm{m}$. drHF: rubbing application in a circular pattern on the skin, agHF: rubbing application against the direction of hair growth, and alHF: rubbing application along with the direction of hair growth. (Color figure can be accessed in the online version.) tion depths in HFs after rubbing. Figure 1 shows the effect of rubbing action and direction on the distribution depth of Fluoresbrite ${ }^{\circledR}$ in the HFs. A slight fluorescence derived from Fluoresbrite $^{\circledR}$ was observed in the shallow region in the open area of HFs and on the SC in the without-rubbing control (Fig. 1a). On the other hand, a bright fluorescence distribution was detected in the deeper region in HFs after agHF action (Fig. 1c) compared with the other rubbing treatments. Rubbing application with drHF (Fig. 1b) and alHF (Fig. 1d) increased fluorescence distribution in the shallow layer of the SC and the open area of HFs compared with the without-rubbing control.

In Vitro Skin Permeation with Rubbing The effect of the rubbing direction on $Q$ was determined using an in vitro skin permeation experiment. Figure 2 shows the skin permeation profiles of CAF through full-thickness skin with or without rubbing action. $Q$ at $120 \mathrm{~min}$ after application $\left(Q_{120 \mathrm{~min}}\right)$ in the without-rubbing control had the lowest value compared

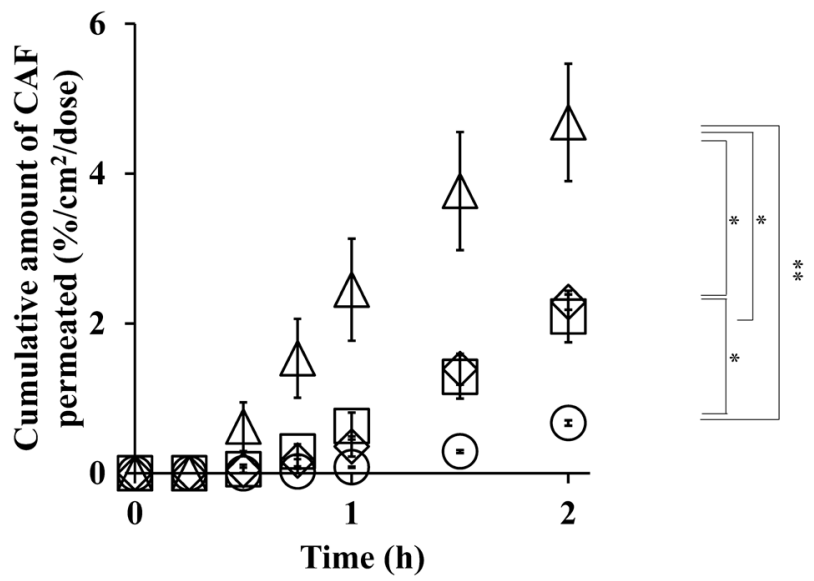

Fig. 2. Time-Course of the Cumulative Amount of CAF That Permeated through Skin after Application with- or without-Rubbing Action

Symbols; $\bigcirc$ : without application, $\square$ : drHF, $\triangle$ : agHF, $\diamond$ : alHF. Each value shows the mean \pm S.D. $(n=3){ }^{*}: p<0.05$ and $* *: p<0.01$
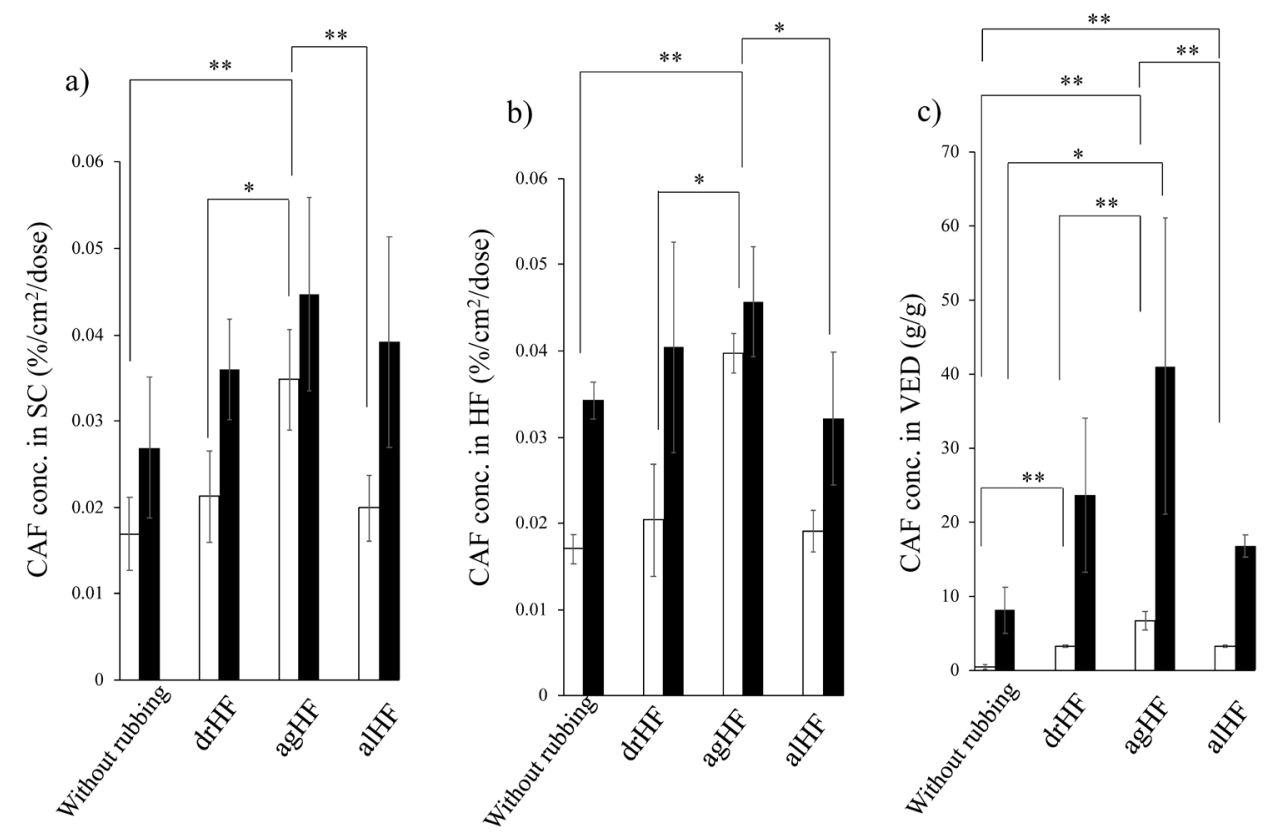

Fig. 3. CAF Concentration in the SC (a), HFs (b), and VED (c) 5 min (Open Column) and 120 min (Closed Column) after Application with- or without-Rubbing Action 


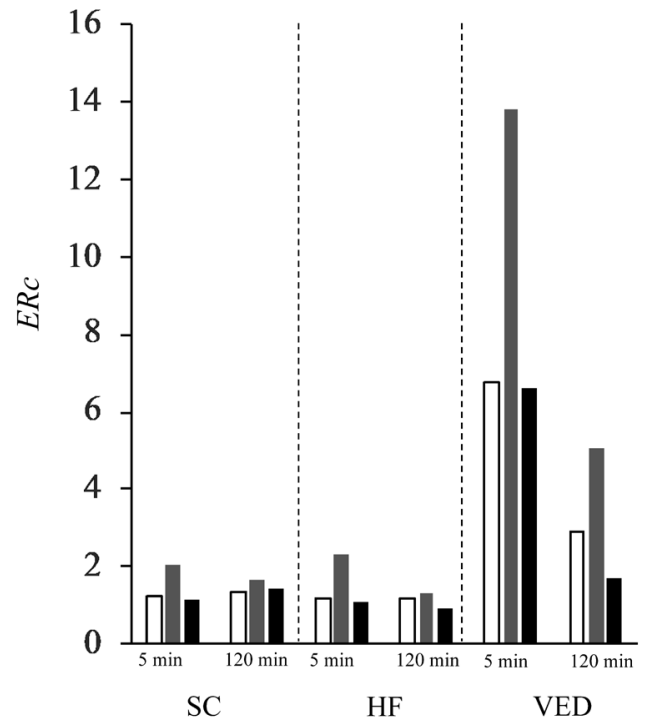

Fig. 4. ERc Values for CAF Concentration in the SC, HFs, and VED 5 and $120 \mathrm{~min}$ after Application

$E R c$ values were calculated using the CAF concentration in the SC, HFs, and VED obtained 5 and $120 \mathrm{~min}$ after application without-rubbing action. White, gray, and black indicate drHF, agHF, and alHF, respectively.

with rubbing actions. Significantly higher $Q_{120 \min }$ values were observed $(p<0.05)$ with the use of rubbing. When $Q_{120 \text { min }}$ values were compared among the rubbing treatments, the following order was obtained: agHF $\left(4.60 \pm 0.78 \% / \mathrm{dose} / \mathrm{cm}^{2}\right.$, mean \pm standard deviation (S.D.), $n=3)>$ alHF $(2.28 \pm 0.10 \%$ / dose $\left./ \mathrm{cm}^{2}, n=3\right)>\operatorname{drHF}\left(2.09 \pm 0.343 \% / \mathrm{dose} / \mathrm{cm}^{2}, n=3\right)$. ERps calculated using $Q_{120 \min }$ versus the without-rubbing control were 7.0-fold for agHF, 3.4-fold for alHF, and 3.1-fold for drHF. On the other hand, decreased ERps were observed when calculated for $Q_{480 \min }$ (2.9-fold for agHF, 1.6-fold for alHF, and 1.9-fold for drHF).

CAF Dispositions in Skin Figure 3 shows the CAF concentrations in the SC, HFs, and VED at 5 and $120 \mathrm{~min}$ after with- and without-rubbing actions. The CAF concentrations at each skin part were increased by passing the application period. In particular, the CAF concentration in the VED was dramatically increased at any application manner.

When CAF concentrations in the SC, HFs, and VED were compared with- or without-rubbing actions, significantly higher CAF concentrations were obtained in the SC, HFs, and VED after rubbing for $5 \mathrm{~min}$. In particular, agHF application showed significantly higher CAF concentrations at each skin part.

Figure 4 shows change in the ERcs of CAF concentration at the SC, HFs, and VED by increasing the application period. Higher ERcs were observed in the VED compared with those in the SC and HFs for each application period. When the $E R c$ s were compared between application periods, the value decreased with the application time.

Figure 5 shows the relationship between ERp and ERc values. The CAF concentrations in the $\mathrm{SC}$ and $\mathrm{HFs} 120 \mathrm{~min}$ after agHF action were higher than those without-rubbing action. However, the ERcs were only 1.66- and 1.33-fold in the SC (shown as in Fig. 5) and HFs (shown as $\boldsymbol{\Delta}$ in Fig. 5), respectively. On the other hand, ERc calculated from the CAF concentration in the VED was about 5.0-fold (shown as $\mathbf{D}$ ) and the $E R c$ value of the skin concentration of CAF, calcu-

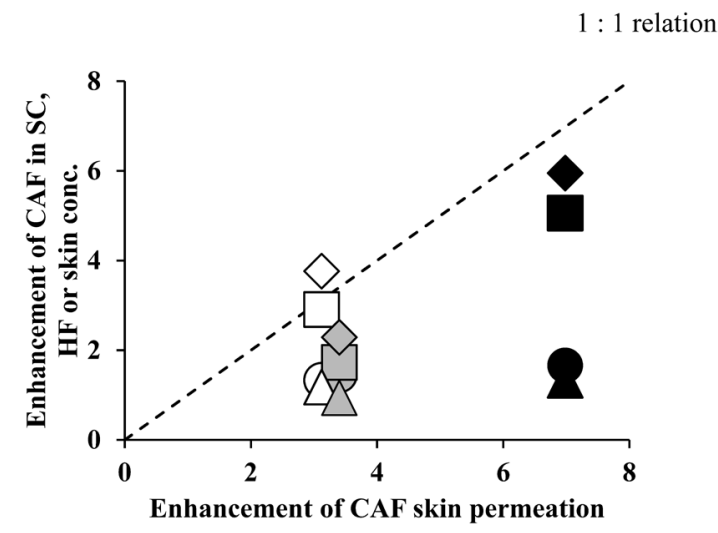

Fig. 5. Relationship between $E R p$ and $E R c$ Values

Dots line: $1: 1$ slope. Symbols: $\bigcirc$ : SC conc, $\triangle$ : HF conc., $\square$ : VED conc., $\diamond$ : total skin conc. White, gray, and black indicate drHF, agHF, and alHF, respectively. The total skin concentration was calculated by dividing the total amount of CAF in the skin by the weight of the skin. White, gray and black indicate drHF, agHF, and alHF, respectively.

lated by dividing the total amount of CAF in the skin by the skin weight, was almost the same as that obtained from skin permeation (shown as $\bullet$ in Fig. 5, 6.0-fold). The same tendency was observed in the other rubbing actions.

\section{Discussion}

Skin with cartilage was used to evaluate the CAF concentration in skin, whereas excised skin was used to perform the in vitro permeation experiment. In our previous report, the CB method was applied to skin with cartilage because Knorr et $a{ }^{10}{ }^{10}$ reported that the follicular reservoir of porcine ear skin remains constant after removal of the ears from cadavers. Although Patzelt et al. ${ }^{11)}$ speculated that the elastic fibers surrounding HFs contracted with in vitro experiments using excised human skin, the CAF concentration in skin was the same with or without cartilage in our preliminary study (data not shown). Thus, different experimental conditions were applied to evaluate skin permeation and skin concentration of CAF.

Enhanced skin permeation has been reported with the use of rubbing application. ${ }^{1-4)}$ Such rubbing during application altered the superficial skin layer, resulting in a faster initial diffusion of topically applied drugs through the SC. ${ }^{12)}$ However, few studies have reported the reasons for the acceleration of drug permeation through skin after topical application with or without rubbing.

Although rubbing conditions and processing period were found to affect the skin permeation of applied drugs, no studies observed the effect of rubbing direction on the skin penetration-enhancement effects. Therefore, in the present experiment, we investigated the effects of rubbing direction on the skin permeation and disposition of CAF.

Fluoresbrite ${ }^{\circledR}$ was distributed at deeper sites in HFs only 5 min after rubbing actions. Because the $\mathrm{CB}$ method can collect drugs in HFs up to approximately $175 \mu \mathrm{m}$ from the SC surface, ${ }^{10}$ measurement of drug concentration in VED was necessary to clarify the skin penetration-enhancement effect by rubbing action.

The $Q_{120 \min }$ values after rubbing of CAF solution were significantly higher than that obtained without-rubbing action. The ERp in skin permeation was about 7.0-fold by agHF 
against without-rubbing action (Fig. 2). Almost the same ERc was observed in the skin concentration of CAF (Fig. 5 shown as $\mathbf{\square}$ ), suggesting that increased VED concentration after agHF contributed to increased skin permeation.

As shown in Fig. 1, the disposition of Fluoresbrite ${ }^{\circledR}$ in the deeper site of the HF was remarkably increased with the use of rubbing action. In the general skin penetration process, distributed drugs in the SC and HF diffuse and permeate through the dermis according to Fick's second law of diffusion. A higher CAF distribution at a deeper site of the HF (dermis site) occurred after a short time with rubbing action. Thus, a higher $E R c$ value in VED was observed $5 \mathrm{~min}$ after application, whereas the ERc was decreased 120 min after application due to an increase in CAF in the VED that diffused from the superficial depth of the SC and HF.

In the present study, porcine ear skin was used to evaluate the effect of rubbing action on CAF permeation through the skin. The diameters of human infundibula are 78, 66, and $170 \mu \mathrm{m}$ for the forearm, forehead, and calf, respectively. ${ }^{6}$ ) Although Jacobi et al. reported that porcine ear skin has similar diameter as the infundibular orifice on human calf, and the diameter of hair shafts on porcine ears is two to five times larger than that of human vellus hair. ${ }^{12}$ ) Therefore, due to the larger diameter of infundibular orifice size in pig skin, the effect of rubbing action observed in porcine skin might be overestimated compared with that observed for the human forearm and forehead.

Rubbing actions increased the skin permeation as well as the skin concentration of CAF. It is considered that the diameter of infundibula orifices changed as a result of stretching the skin due to the rubbing action. In particular, the diameter might be opened more by agHF because the process involved hairs being rubbed in the opposite direction to the flow of the hairs. Thus, opening of infundibula orifices caused by changes in hair flow may be a reason for the greater enhancement effect by agHF. Rubbing action with alHF and agHF may also change the diameter of the infundibula orifices. However, unlike rubbing application with agHF, these processes are less effective for the increased skin permeation and skin disposition of CAF compared with that with agHF. Rubbing actions with drHF seemed to be slightly higher than that with alHF. This was thought to be related to rubbing action with drHF containing some parts of the agHF and alHF processes.

The predominant permeation pathways of hydrophilic and lipophilic drugs are HFs and the SC, respectively. ${ }^{13-15)}$ In our previous report, ${ }^{10)}$ the concentrations of CAF and a lipophilic drug, 4-butylresorcinol (M.W: $\left.166.2, \log K_{\mathrm{o} / \mathrm{w}}: 2.85\right)$, in HFs were measured using the $\mathrm{CB}$ method. Although the predominant permeation pathway of CAF was HFs, the 4-butylresorcinol concentration in HFs was higher than that for CAF due to their different lipophilicities. ${ }^{10)}$ In the present study, no lipophilic drugs were examined, but rubbing application may enhance drug disposition in the skin independently its lipophilicity by physically opening of the funnel shaped HFs. In addition, the distribution of fluorescently labeled nanoparticles, Fluoresbrite ${ }^{\circledR}$, was enhanced by rubbing application in the present study, suggesting that the HF distribution of high molecular weight of compounds as well as vehicles such as liposomes and micelles may be enhanced by rubbing actions.

There are several types of topical formulations; gels, ointments, lotions, and patches, etc. The enhancement effect of the skin permeation of drugs by rubbing may vary according to the type of formulation applied. Liquid and semisolid type formulations easily change their shape by the rubbing action on skin. Therefore, high skin permeation of drugs from these formulations should be observed after rubbing.

Further experiments will be necessary to clarify the skin penetration-enhancement effect by rubbing application; permeation experiments using different sites (face, scalp, and chest) of human skin, the effect of different physicochemical properties of topical formulation, and the effect of lipophilicity and/or molecular weight of drugs on the enhancement by rubbing application.

\section{Conclusion}

Rubbing action enhanced the skin permeation of topically applied CAF, and the direction of the rubbing action affected the skin permeation. Although further experiments should be performed, this experiment provides useful information on the effect of rubbing action and rubbing direction on the skin permeation of topically applied drugs.

Conflict of Interest The authors declare no conflict of interest.

\section{References}

1) Ishii H., Todo H., Sugibayashi K., Biol. Pharm. Bull., 33, 876-880 (2010).

2) Nguyen H. X., Puri A., Banga A. K., Int. J. Pharm., 519, 22-33 (2017).

3) Hasler-Nguyen N., Fotopoulos G., BMC Res. Notes, 5, 321 (2012).

4) Nguyen H. X., Puri A., Banga A. K., Int. J. Pharm., 519, 22-33 (2017)

5) Shah V. P., Am. J. Clin. Dermatol., 2, 275-280 (2001).

6) Otberg N., Richter H., Schaefer H., Blume-Peytavi U., Sterry W., Lademann J., J. Invest. Dermatol., 122, 14-19 (2004).

7) Scheuplein R. J., J. Invest. Dermatol., 48, 79-88 (1967).

8) Trauer S., Patzelt A., Otberg N., Knorr F., Rozycki C., Balizs G., Büttemeyer R., Linscheid M., Liebsch M., Lademann J., Br. J. Clin. Pharmacol., 68, 181-186 (2009).

9) Blume-Peytavi U., Massoudy L., Patzelt A., Lademann J., Dietz E., Rasulev U., Garcia Bartels N., Eur. J. Pharm. Biopharm., 76, 450-453 (2010).

10) Abe A., Saito M., Kadhum W. R., Todo H., Sugibayashi K., Int. J. Pharm., 542, 27-35 (2018).

11) Patzelt A., Richter H., Buettemeyer R., Huber H. J. R., BlumePeytavi U., Sterry W., Lademann J., Eur. J. Pharm. Biopharm., 70, 234-238 (2008).

12) Jacobi U., Kaiser M., Toll R., Mangelsdorf S., Audring H., Otberg N., Sterry W., Lademann J., Skin Res. Technol., 13, 19-24 (2007).

13) Knorr F., Lademann J., Patzelt A., Sterry W., Blume-Peytavi U., Vogt A., J. Pharm. Biopharm., 71, 173-180 (2009).

14) Todo H., Kimura E., Yasuno H., Tokudome Y., Hashimoto F. Ikarashi Y., Sugibayashi K., Biol. Pharm. Bull., 33, 1394-1399 (2010).

15) Liu X., Grice J. E., Lademann J., Otberg N., Trauer S., Patzelt A., Roberts M. S., Br. J. Clin. Pharmacol., 72, 768-774 (2011). 